\title{
A method to analyze adaptive optics images of binary stars
}

\author{
O. Bendinelli ${ }^{1}$, D. Bonaccini ${ }^{2}$, S. Falsini ${ }^{1}$, and G. Parmeggiani ${ }^{3}$ \\ 1 Dipartimento di Astronomia, Universitá di Bologna, Via Zamboni 33, 40050 Bologna, Italy \\ e-mails: bendinelli@bo.astro.it, falsini@bo.astro.it \\ 2 European Southern Observatory, Karl-Schwarzschild-Str. 2, D-85746 Garching bei Muenchen, Germany \\ e-mail: dbonacci@eso.org \\ 3 Osservatorio Astronomico di Bologna, Via Zamboni 33, 40050 Bologna, Italy \\ e-mail: parmeggiani@bo.astro.it
}

Received December 15, 1997; accepted October 2, 1998

\begin{abstract}
We present a fast and robust ad-hoc method to analyze a set of short exposure images of a close binary star taken by the adaptive optics system of the ESO $3.6 \mathrm{~m}$ telescope. At first each image is fitted, assuming as a radially symmetrical approximation of its "instantaneous" PSF, the superposition of a central Gaussian and of a Moffat torus. Owing to the coral variability and asymmetry of the PSF the fit gives a poor estimate of the component's luminosity ratio; nevertheless it reproduces satisfactorily the global structure of the PSF and above all gives the accurate sub-pixel positions of the two stars. This allows to obtain, by the shift-and-add procedure, the mean set image from which the final parameters of the two stars and the mean PSF during the observing run are derived. The results from a set of AO images of the close binary system $\tau$ Canis Maioris are consistent with those previously obtained by one of us using the iterative blind deconvolution. Extensive simulations give evidence that the magnitude difference and the separation of the components can be estimated within about 0.05 mag and 0 .'005 respectively when the secondary star image does not overlap a bright artifact of the AO system.
\end{abstract}

Key words: stars: $\tau$ Canis Majoris — instrumentation adaptive optics; techniques: image processing

\section{Introduction}

Ground-based telescopes using Adaptive Optics (AO) are now able to acquire images with a diffraction limited resolution.

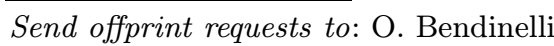

The AO systems realize the out-running idea of Babcock (1953) to compensate in real-time the atmospheric turbulence effects by suitable fast deformations of the optics. The exhaustive treatment of the AO subject in its various facets and an extensive list of relevant works can be found in the monograph by Tyson (1991) and in the review by Beckers (1993).

To improve the information content of a set of AO images it is necessary to deconvolve them from the Point Spread Function (PSF) as usual, and this is even more needed since each image, as well its PSF, is characterized by a variable amount of compensation. Thus the main problem faced when analyzing AO images, is the determination of the instantaneous PSF of the optical system during the acquisition. The PSF is affected by the diffraction pattern of the telescope, by residual static aberrations and by the AO servo-loop correction. Static aberrations can have a slow (tens of minutes) variability in time, due to optomechanic flexures and thermal effects. The servoloop residuals instead have rapid variability induced by short (seconds) and long (minutes or tens of minutes) seeing changes, see for instance Rigaut et al. (1991), Tessier et al. (1994), Christou \& Bonaccini (1996a).

It has been found experimentally that in general "short" exposure IR images (some hundredth of seconds for sufficiently bright sources) have a larger variability in the PSF, while "long" exposure images (from some seconds to some minutes) benefit from a more stable PSF. During AO observations, to apply deconvolution, the PSF of the system has to be retrieved either from a point source within the field, or from an offset point source observed separately (usually called the PSF calibrator star). Methods to derive the PSF from the AO wavefront sensor (WFS) signal have been proposed and proven successful under certain conditions of SNR (e.g., Véran et al. 1997).

In all of these cases however, the PSF is determined with limited accuracy. Either because it has been 
measured at a different time, at a different position within the field and with different fluxes on the AO wavefront sensor, or because (in the case of WFS retrieval) it does not detect small scale artifacts of the image. In general the PSF is formed by a central core with Airy rings, superimposed on an extended and sometimes irregular halo. In the case of the ESO ADONIS adaptive optics system, in use at the $3.6 \mathrm{~m}$ telescope at La Silla, several bumps on the PSF halo are present with static and dynamic components. In the images we examine in this paper, the three brightest bumps of variable intensity $(0.5-1 \%)$ and position, due to uncompensated triangular coma aberration, roughly individuate the deformed first Airy ring.

Hence to recover the image, methods allowing the use of a relaxed or "best fitted" PSF in the deconvolution are necessary as the PSF is not accurately measured. This is the case of the Iterative Blind Deconvolution (IBD) technique, which yields at the same time the best fitted PSF and image. But deconvolution belongs to the class of unstable inverse problems, i.e., the solution by any classical method of the linear system discretizing the convolution integral equation is not continuously dependent from the data even if the PSF is exactly known. Thus every efficient numerical method of deconvolution in order to reach an approximate and stable solution must use statistical or deterministic constraints on the solution itself (such as non negativity and smoothness, maximum likelihood or maximum entropy, minimum norm and so on, see, e.g., Lucy 1994) and these constraints necessarily involve a controllable amount of bias in the solution; see for instance Titterington (1985) or Bendinelli et al. (1986). For IBD to work, since the PSF is less defined, stronger constraints are required. These are imposed not only on the solution (as in the usual deconvolution with well known PSF) but also on the PSF itself. They are based on a priori knowledge or well founded hypotheses, as pointed out by Jefferies \& Christou (1993) or Thiebaut \& Conan (1995).

The IBD technique has been successfully applied by one of us to Adonis AO data, using the IDAC program developed by Matt Chesalka and Keith Hegge at Steward Observatory. This algorithm starts from a good estimate of the PSF, and not from a blind guess of it, and yields at the same time the best fitted PSF for each frame, and the image. This method has been tested on point sources and on extended objects, with simulations and real case data (e.g., Christou \& Bonaccini 1996b, 1997). It is however slow in converging, and not necessarily the more efficient software for all astrophysical observations with AO.

The aim of this work is to show that when dealing with a set of AO images of a close binary star, the magnitudes and the positions of their components may be derived in a faster and more direct way than with IBD.

The method we present is based on a two stage procedure which at first gives photometry and separation of the components by a non linear fit, then gives the detailed PSF by deconvolution of the observed images from the sum of the two delta functions which represents the true intensity distribution.

It has been applied to 3 sets of 48 images each (in the $J, H$ and $K$ bands respectively) of the binary star $\tau$ Canis Majoris $(\tau \mathrm{CMa})$, which were taken with ADONIS at the ESO $3.6 \mathrm{~m}$ telescope during a technical run. Each set is composed by the first 48 of a series of 200 short exposure images $\left(t_{\text {exp }}=0.05 \mathrm{~s}\right.$, time step $\left.0.74 \mathrm{~s}\right)$ already analyzed by Christou \& Bonaccini (1996b, 1997) by means of IBD using IDAC.

This case is a particularly difficult one, as the companion is located on the first Airy ring of the $H$-band image, and difficult to disentangle from triangular coma features.

\section{Binary star AO image analysis}

If within the isoplanatic field ( $n \times n$ pixels wide) there is only a close binary star, the observed short image is the superposition of two shifted and unnormalized nearly instantaneous PSFs or, in other words, the convolution of the PSF with two unnormalized impulse distributions. When the PSF is not known and not radially symmetric, the successful analysis of the image data would give positions and luminosities of the two stars and the PSF intensity in each pixel (i.e., a total of $6+n^{2}$ values); thus, whatever the approach followed, an approximate estimate of these values can be obtained only by using prior knowledge or suitable hypotheses (in definitive solution constraints) to overcome the initial lack of information. The availability of a set of short images does not conceptually improve the situation, owing to the PSF time-space variability and the matching effects of residual image motion (see Christou \& Bonaccini 1997) and pixel size, which in the actual case is about half of the Airy disk. Since the pixel intensity is an integrated value, really small structures such as the cores of the two stars or the bumps due to the triangular coma can appear somewhat different from image to image, even with very similar PSFs, if their centers do not coincide with the center of the corresponding pixel of relative maximum intensity, and the derived photometry can be misleading. But in practice these drawbacks are compensated for sufficiently by the use of statistical tools, because the set of $\mathrm{N}$ short images can be regarded as a large random sample whose variability is due to many unknown combining effects (the instantaneous parameters of the atmospheric turbulence, the fine reponse of the AO system, etc.). The first step of our procedure is the fit of each short image as a superposition of two weighted radially symmetric approximations of the PSF. This yields an estimate of the position and intensity of the two stars, as well as of the PSF's global shape and of the partition of its light between the 3 characteristic zones: the central core, the defaced first diffraction ring and the extended halo. At this stage it is possible to obtain either the features of the binary system by averaging the intensity and position parameters, or the set of the 
detailed nearly instantaneous PSFs by deconvolution of each short image from its joint two-impulse distribution. But the short PSF set thus obtained (and used below in Sect. 4) shows the expected large variability, and hence is scarcely useful to understand the performances of the AO system during the observations, while the scatter of the parameters, which will be discussed below, enables an accurate determination only of the binary separation, not of the components' intensity ratio. Thus, in the second step we obtain the set's mean image by shift-and-add of the short images, using for each of these, as shifting parameters, the coordinates of the center of the primary star. Then the mean image is fitted as the short images in the first step, yielding the adopted features of the binary system (relative position and luminosity of the components) and the azimuthally averaged behaviour of the set's mean PSF, while its detailed bidimensional structure is derived by deconvolution of the mean image from the two-impulse distribution with the suitable parameter values.

\subsection{Short image parametrization}

To fit the $\mathrm{N}$ short images of the double star we use as a smooth approximation of the instantaneous PSFs (denoted as $p_{k}(x, y)$, with $\left.k=1,2, \ldots, N\right)$ the sum of a centered Gaussian and of a Moffat's torus with a core parameter $R_{\mathrm{c}}$ and the same radius $R_{\mathrm{t}}$ of the first diffraction ring of the ESO 3.47 meter telescope, which has a 0.479 obstruction factor, as given by the Lyot stop inside the IR camera. It must be stressed that the "ad hoc" circular approximation of the PSF, inappropriate e.g. to represent the PUEO star images (Rigaut et al. 1991), was chosen after some trial on the more suitable fitting function. In particular, we have first used the sum of a central Gaussian, a Gaussian torus with free radius and an exponential. But the behaviour of the wings of a short PSF in mean better belongs to a power law, (see Fig. 6), and the torus radius was found to be very close to that of the diffraction ring in almost all cases, thus its estimate was left out. The adopted expression of the PSF (containing the four parameters $a_{1}, \sigma, R_{\mathrm{c}}$ and $\beta$ ) explicitly reads as

$$
\begin{aligned}
p_{k}(x, y)= & a_{1} \mathrm{e}^{-\left(x^{2}+y^{2}\right) / 2 \sigma^{2}}+ \\
& a_{2} K\left[1+\left(\sqrt{\left(x^{2}+y^{2}\right)}-R_{\mathrm{t}}\right) / R_{\mathrm{c}}\right]^{-\beta}
\end{aligned}
$$

where $a_{2}=1-a_{1}$ and the normalizing factor $K$ of the torus must be evaluated by a numerical integration, because this cannot be done analytically. It is readily apparent that the behaviour of Eq. (1) mimics a deformed diffraction pattern showing the remainders of the central maximum and of the first ring, but with a large fraction of the total light $(>50 \%$ ) falling in an extended, roughly circular halo. When the short PSF is characterized by the bumps due to the uncompensated triangular coma, as in the present case, the extension of Eq. (1) to represent also these features would contain many more non linear parameters, whose estimate, though possible in principle, is very difficult in practice. Above all, this extension should be of interest if the primary goal of the analysis were the search of an accurate analytical approximation of the PSF, not the differential astrometry and photometry of a binary star. In our opinion, also, the effects and limitations on the AO image analysis caused by the said bumps are less important than generally believed because they contain only a few percent of the total light, are located close to the first Airy ring, and are more irregular and extended in shape than the round and spiky core of a star image produced by an AO system. Also, it must be pointed out that owing to the amount of light in the PSF halo the image of a close binary is elongated along the centers' joining line, if the secondary luminosity is not too low, with quite a uniform distribution of the outer light; the strongest bumps of the primary are well visible, while those of the secondary progressively merge into the primary light as they become fainter. In conclusion, the discrimination of the core of the secondary star from one of the bumps of the primary seems possible by means of a visual inspection (see e.g. Figs. 1 and 2) or by an expert program, with the exception of the critical case in which the two features are very close or overlap. When this occurs the image must be still elongated, but the features separation is not very reliable. The only way to study such a binary star is a previous rotation of the $\mathrm{AO}$ system, if technically possible, so that these features do not overlap. The dependence of the AO image of a binary system on the values of position and intensity of its secondary star will be again considered in the section on tests and numerical simulations, as well as the influence of the choice of the secondary core among the other bright features surrounding the primary center. Using the PSF approximation of Eq. (1), the intensity distribution $i_{k}(x, y)$ in a short image (after the usual pre-processing of CCD data, background subtraction and neglecting the pixel integration) reads as

$i_{k}(x, y)=L_{\mathrm{t}}\left[l_{1} p_{k}\left(x-x_{1}, y-y_{1}\right)+\right.$

$$
\left.l_{2} p_{k}\left(x-x_{2}, y-y_{2}\right)\right]
$$

where $L_{\mathrm{t}}$ is the known total image intensity, $l_{1}$ and $l_{2}$ (with $l_{1}+l_{2}=1$ ) are the components' relative intensities, and $x_{1}, y_{1}, x_{2}, y_{2}$ the centers. To estimate the nine nonlinear parameters in Eq. (2) (i.e.: $a_{1}, e_{1}, \sigma, R_{\mathrm{c}}, \beta, x_{1}, y_{1}, x_{2}$ and $\left.y_{2}\right)$, we use the Newton-Gauss regularized method (Bendinelli et al. 1987, hereinafter NGR), but nearly the same result could be obtained by any current non-linear fitting routine. The implemented procedure improves the classical linearization by using the Moore-Penrose pseudo inverse of ill-conditioned matrices (see Penrose 1956) and Tikhonov first-order regularization (see Tikhonov \& Arsenin 1977), so that all parameters usually converge at $0.5 \%$ level in less than 10 iterations, starting from a suitable set of initial parameters, chosen as follows. For all the short images we adopted the same starting values 


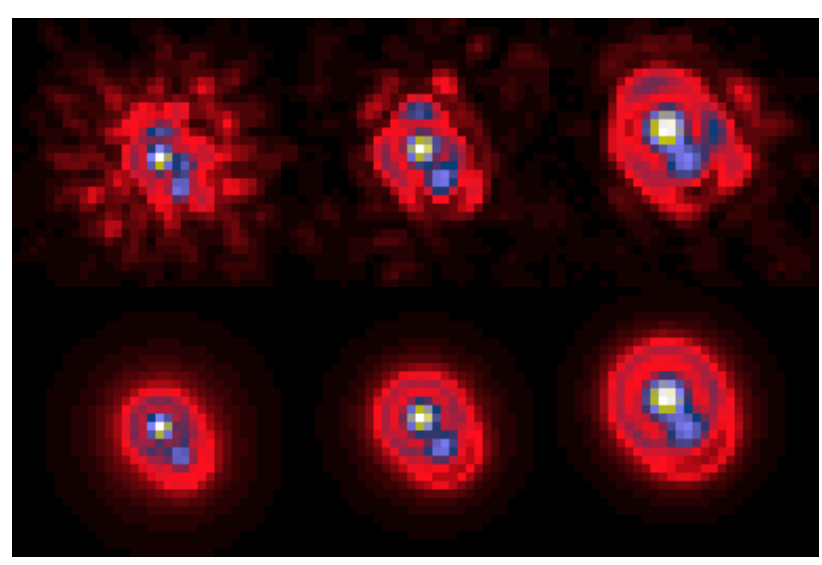

Fig. 1. Good $J, H$ and $K$ short exposure images of $\tau$ CMa (first row), and their fit by Eq. (1) (second row). Parameter values in Table 1

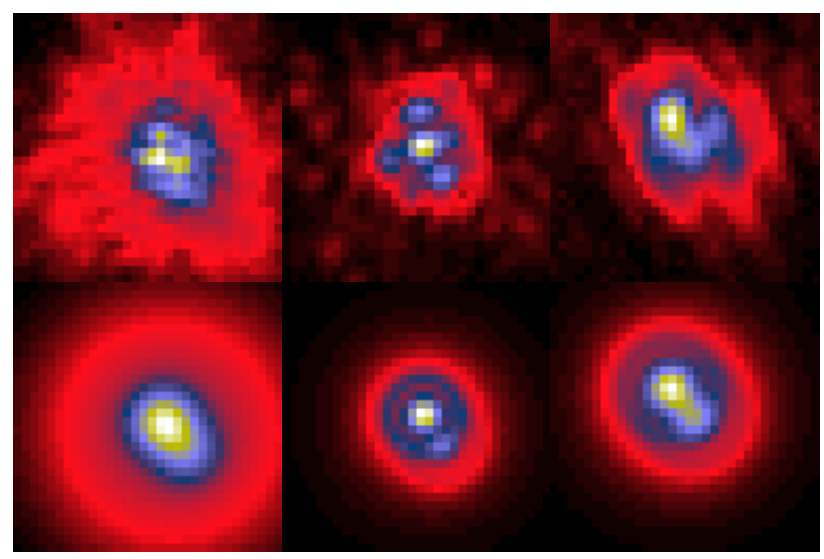

Fig. 2. $\operatorname{Bad} J, H$ and $K$ short exposure images of $\tau$ CMa (first row), and their fit by Eq. (1) (second row). Parameter values in Table 1

$l_{1}=0.7 L_{\mathrm{t}}, a_{1}=0.3 L_{\mathrm{t}}, \beta=1$, on the grounds of some preliminary fits of a few good and bad images, i.e., with the relevant details more or less stable both in position and luminosity as clearly shown in Figs. 1 and 2 (top). The pairs $\left(x_{1}, y_{1}\right)$ and $\left(x_{2}, y_{2}\right)$ were instead taken as the coordinates of the maximum intensity pixels in the unmistakable primary core and in the assumed secondary one. Finally, the band-dependent value of $\sigma$ was obtained by a bilinear interpolation of the intensity of the pixels surrounding the center of the primary star, and $R_{\mathrm{c}}$ was always taken equal to $\sigma$.

The usefulness of Eq. (2) to obtain a good smooth approximation of the intensity distribution in short images is shown in Figs. 1 and 2 (bottom). The correlation coefficients $c$ between observed and parametrized images, which are reported in Table 1 with the relevant fitted features of the model, strongly prove the procedure's reliability. But the behaviour of the fitting parameters and of the

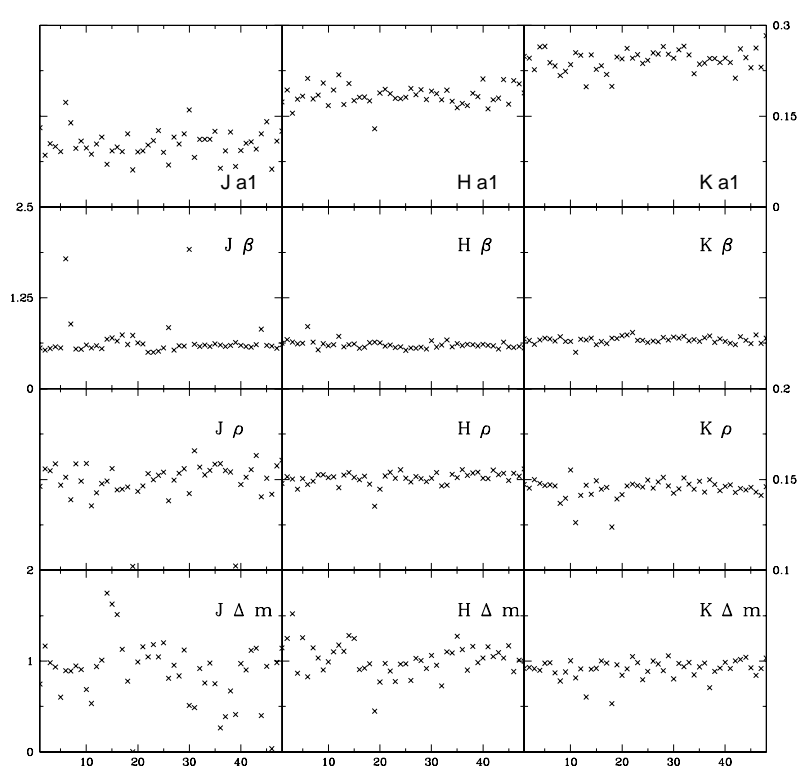

Fig. 3. Dependence of derived features of the binary system in the $J, H, K$ bands along the set's image sequence

derived binary system features along the image sequence must be pointed out, recalling that all the obtained values have bias components which are very hard to quantify, generated either by the numerical stabilization of the non-linear fit, or by pixel integration and residual image motion effects. The scatter of the parameters related to the PSF shape (see for instance the sets of $a_{1}$ values in Fig. 3) is mainly due to its well known short-time scale variability and wavelength dependence. The apparent deplacement of the centers' coordinates, instead, follows the residual image motion, which is the same in any point of the isoplanatic field; thus the binary components' separation is really the quantity best estimated by the fit (see Fig. 3 and Tables 1 and 2) in good agreement with the previous result of $\rho=0$ "' 151 obtained by Hipparcos (see The Hipparcos and Tycho Catalogues 1997). The estimate of the components' luminosity ratio in a close binary system worsens as the secondary becomes fainter, because its halo and the diffraction features merge into the primary halo, while the secondary core, the only detail still visible, becomes more contaminated and thus the fitting parameters more uncertain and dependent on the fine structure of the PSF halo. The lovering of the compensation degree acts in a similar way since it decreases the central core's intensity and yields a more irregular light distribution in the intermediate PSF zone, thus making the analytical approximation used less adequate. Finally, it must be stressed that the actual position of the secondary star can strongly twist the fitting parameters, particularly when it falls near the defaced first diffraction ring or, even worse, if it overlaps one of its bumps. In these cases the secondary 
Table 1. Binary star features by fit of images in Figs. 1 and 2

\begin{tabular}{ccccccccc}
\hline & & $\Delta_{\mathrm{m}}$ & $\Delta_{\mathrm{s}}$ & $\sigma$ & $R_{\mathrm{c}}$ & $\beta$ & $L_{\mathrm{g}}$ & $c$ \\
\hline$J$ & $\mathrm{~g}$ & 1.0449 & 0.1523 & 0.8467 & 0.5282 & 0.5157 & 0.1263 & 0.97 \\
& $\mathrm{~b}$ & 0.8932 & 0.1512 & 2.2977 & 13.1249 & 1.7858 & 0.1726 & 0.97 \\
$H$ & $\mathrm{~g}$ & 1.0356 & 0.1507 & 1.0690 & 0.6491 & 0.6090 & 0.2115 & 0.98 \\
& $\mathrm{~b}$ & 1.5202 & 0.1502 & 1.2082 & 0.8849 & 0.6421 & 0.1547 & 0.96 \\
$K$ & $\mathrm{~g}$ & 1.0319 & 0.1460 & 1.3508 & 0.7931 & 0.6945 & 0.2828 & 0.99 \\
& $\mathrm{~b}$ & 0.6050 & 0.1468 & 1.7388 & 1.2921 & 0.6691 & 0.1985 & 0.97 \\
\hline
\end{tabular}

Note: g good image, b bad image.

luminosity derived by the fit of an image may result either underestimated or overestimated, as seems to be confirmed by the conflicting magnitude difference values reported in Table 1 , recalling that the binary separation is very close to $R_{\mathrm{t}}$ in the $H$ band. In conclusion, the fit of short images gives a good global radially symmetric approximation of the instantaneous PSFs and quite a satisfactory estimate of the stars' positions, but a poor differential photometry, as occurs in the interferometry of double stars (see for instance Ten Brummelaar et al. 1996). Averaging the fit values, whose large scatter is due either to PSF variability or to non-linear numerical effects, there is a slightly improvement in the photometry (see Table 2), while a larger one seems attainable by neglecting the bad images with parameters far from the set mode (see Fig. 3); but the correctness of this procedure should be secured only by the normality of the parameter set.

\section{The mean set image determination}

As explained above, the binary system's instantaneous centers (hence also the residual image motion) are the most reliable parameters derived by fitting the short images. This suggests that the image analysis can be refined by means of the mean set image $i_{\mathrm{m}}(x, y)$ obtained by shiftand-add on multiple frames by using the Fourier shift theorem, i.e.,

$i_{\mathrm{m}}(x, y)=\mathcal{F}^{-1}\left[\frac{1}{N} \sum_{k=1}^{N} I_{k}(u, v) \mathrm{e}^{-2 \pi j\left(x_{k} u+y_{k} v\right)}\right]$

where $j$ is the imaginary unit, $I_{k}(u, v)$, with $k=1,2, \ldots$, $N=48$, is the Fourier transform $(\mathcal{F})$ of the observed image $i_{k}(x, y), \mathcal{F}^{-1}$ is the inverse Fourier transform and the pairs $\left(x_{k}, y_{k}\right)$ are the primary centers found by the above described general fit. When compared to the set images, the mean image is really characterized by a much better known position of the primary center (see Fig. 4), by an improved signal to noise ratio in each pixel, and by a better definition of the relevant details such as the secondary core and the primary bumps.

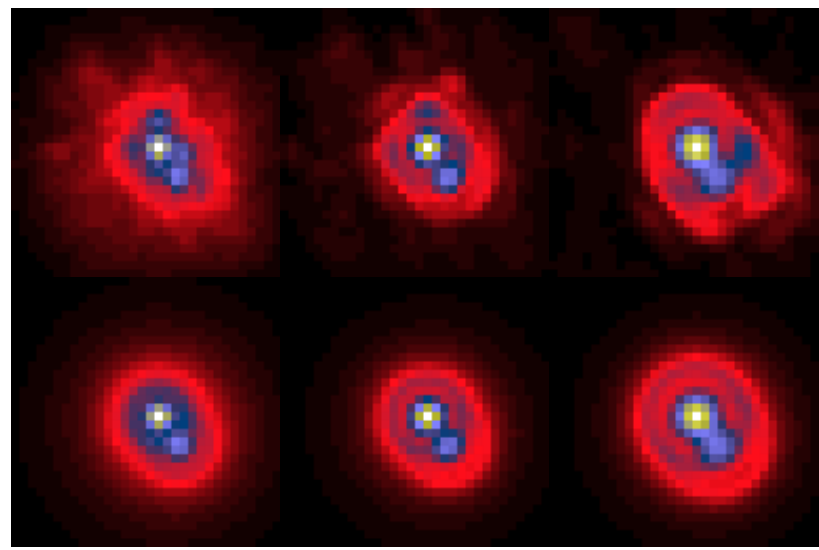

Fig. 4. Mean images in the $J, H$ and $K$ bands of the binary system $\tau \mathrm{CMa}$ (first row) and their fits (second row). The symmetrical light distribution around the centers of the primary component demonstrates the astrometric accuracy attainable by the shift-and-add algorithm using fit position parameters. The correlation coefficient are 0.98, 0.99 and 0.99 , respectively

\section{Results from the mean image analysis}

When the mean image in each band is fitted, yielding at the same time the double system features (see Table 3 and Fig. 4) and the PSF's global structure (Fig. 5, bottom), the results are more reliable than the averages obtained in Sect. 2. This because the fit now uses improved values of the pixel intensities, requires two parameters less than the right-hand side of Eq. (2) (namely $x_{1}$ and $\left.y_{1}\right)$, and starting with the above averages as initial parameters requires lower stabilizing constraints, so that its results are less biased. But the way these are obtained does not allow an easy estimate of their errors, which are conservatively approximated with upper limits equal to the standard deviations in Table 3, although realistic simulations (see Sect. 4) indicate values about half of these. The smooth radial approximation of the mean PSF (see Fig. 5, bottom) is simply obtained by inserting in the right hand side of Eq. (1) the suitable fitting parameters of the mean image. The accurate bidimensional light 
Table 2. Binary star features and PSF paramters for $J, H$ and $K$ sets of images averaging the individual fits

\begin{tabular}{cccccccc}
\hline & $\Delta_{\mathrm{m}}$ & $\Delta_{\mathrm{s}}\left(^{\prime \prime}\right)$ & $\left.\mathrm{PA}^{\circ}\right)$ & $\sigma(\mathrm{pix})$ & $R_{\mathrm{c}}(\mathrm{pix})$ & $\beta$ & $L_{\mathrm{g}}$ \\
\hline$J$ & $0.86 \pm 0.26$ & $0.149 \pm 0.012$ & $-54.5 \pm 6.4$ & $1.04 \pm 0.27$ & $1.58 \pm 0.38$ & $0.66 \pm 0.26$ & $0.104 \pm 0.023$ \\
& $0.96 \pm 0.11$ & $0.151 \pm 0.007$ & $-55.1 \pm 2.3$ & $0.97 \pm 0.13$ & $0.95 \pm 0.29$ & $0.59 \pm 0.05$ & $0.104 \pm 0.012$ \\
$H$ & $1.01 \pm 0.14$ & $0.151 \pm 0.004$ & $-55.8 \pm 2.0$ & $1.18 \pm 0.17$ & $0.88 \pm 0.39$ & $0.60 \pm 0.05$ & $0.184 \pm 0.016$ \\
& $1.02 \pm 0.11$ & $0.151 \pm 0.003$ & $-55.6 \pm 1.0$ & $1.12 \pm 0.07$ & $0.77 \pm 0.14$ & $0.60 \pm 0.04$ & $0.186 \pm 0.014$ \\
$K$ & $0.91 \pm 0.08$ & $0.145 \pm 0.005$ & $-55.0 \pm 3.1$ & $1.47 \pm 0.13$ & $0.99 \pm 0.21$ & $0.67 \pm 0.04$ & $0.242 \pm 0.017$ \\
& $0.93 \pm 0.05$ & $0.146 \pm 0.003$ & $-54.8 \pm 1.9$ & $1.44 \pm 0.09$ & $0.97 \pm 0.18$ & $0.67 \pm 0.04$ & $0.244 \pm 0.014$ \\
\hline
\end{tabular}

Note: first row values, in each band, were derived by averaging all individual fit parameters, second row values by using only good images; quoted errors are standard deviations, recalling that the pixel size is 0. . 035 .

Table 3. $\tau$ CMa features and PSF parameters from fitting the mean set images (first row in each band) and previous results by Christou \& Bonaccini 1996b (last row)

\begin{tabular}{cccccccc}
\hline & $\Delta_{\mathrm{m}}$ & $\Delta_{\mathrm{s}}$ & $\mathrm{PA}$ & $\sigma$ & $R_{\mathrm{c}}$ & $\beta$ & $L_{\mathrm{g}}$ \\
\hline$J$ & 0.92 & 0.152 & -54.9 & 0.97 & 0.89 & 0.58 & 0.099 \\
& 0.79 & 0.150 & -56.0 & & & & \\
$H$ & 1.02 & 0.151 & -55.4 & 1.15 & 0.80 & 0.59 & 0.182 \\
& 0.80 & 0.148 & -57.0 & & & & \\
$K$ & 0.93 & 0.145 & -55.0 & 1.44 & 0.95 & 0.67 & 0.239 \\
& 0.88 & 0.148 & -57.0 & & & & \\
\hline
\end{tabular}

distribution of the mean PSF (Fig. 5, top) is instead derived by deconvolution of the mean image from the pair of impulse functions, with appropriate amplitude and position parameters, which represents the "true" photometric structure of a distant binary system, i.e., is given by

$$
\operatorname{PSF}_{\mathrm{m}}(x, y)=\mathcal{F}^{-1}\left[\frac{I_{\mathrm{m}}(u, v)}{l_{1}+l_{2} \mathrm{e}^{-2 \pi j\left(u\left(x_{2}-x_{1}\right)+v\left(x_{2}-x_{1}\right)\right)}}\right]
$$

where $I_{\mathrm{m}}(u, v)$ and the denominator in the right-hand side are respectively the $\mathcal{F}_{\mathrm{S}}$ of the mean image and of the impulse sum. It must be stressed that the $\mathcal{F}$ of the latter does not have zeroes nor values approaching zero, thus $\mathcal{F}^{-1}$ in Eq. (4) can be performed without the need of any numerical stabilization. The global similarity between the detailed mean PSF and its radially symmetric approximation is clearly shown in Fig. 5, while the local disagreements can be seen in Fig. 6, which displays the actual intensity distribution along the axes in the mean PSF and in its circular approximation. Also, it is worth mentioning that the mean images in Fig. 4 are very similar to good images in Fig. 2, which implies a small occurrence of bad images. Finally, the mean performances of ADONIS during the $\tau$ CMa observations may be easily inferred by comparison (see Fig. 7) of the intensity profile and the encircled energies in the circular PSF approximation with the corresponding values in the diffraction pattern of the ESO $3.6 \mathrm{~m}$ telescope.

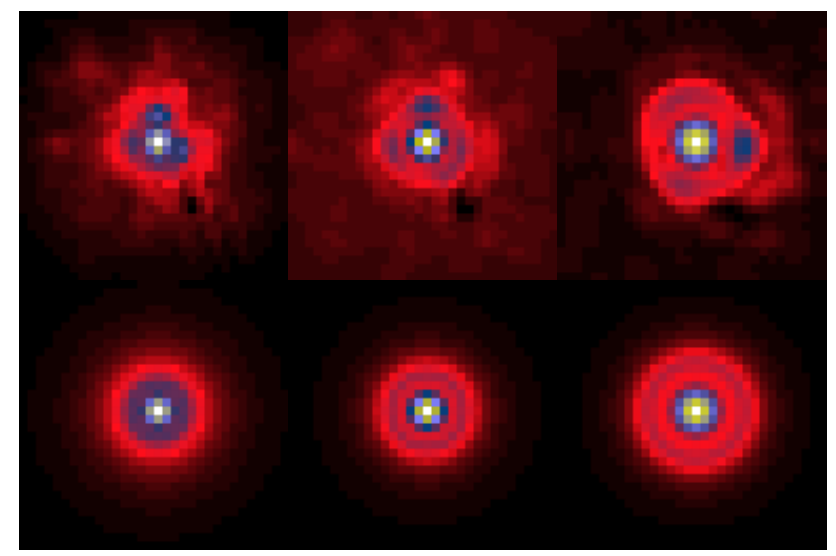

Fig. 5. Mean PSF in the $J, H$ and $K$ bands (first row) and their circular approximation (second row). Note, as in Fig. 4, the accurate centering

\section{Tests and simulations}

The agreement shown in Table 3 between our findings and the previous ones by IBD is even too good in view of the fact that neither method is rigorous. A way to settle which of them gives the more correct results in this particular application should be the comparison with HST results on the $\tau$ CMa system, but so far these are lacking. Our procedure requires much less iterations and CPU occupation than IBD, but also the preliminary identification of the secondary core, thus it is worth checking how it works when the binary star components are characterized by different values of intensity and position. To address the matter we have first used (in the $J, H$ and $K$ bands) a set of 48 simulated images of binary stars, each with a random magnitude difference of the components in the range $1.0-2.5$, random position of the secondary star within a square box of size $3 R_{\mathrm{t}}$ centered on the primary and a random PSF drawn out of the PSF set obtained above by deconvolution of short images from the related two-impulse distribution. For instance, Fig. 8 shows what would be the instantaneous appearance of a binary star, observed with the partially compensated PSF of ADONIS, if its components where characterized by: 

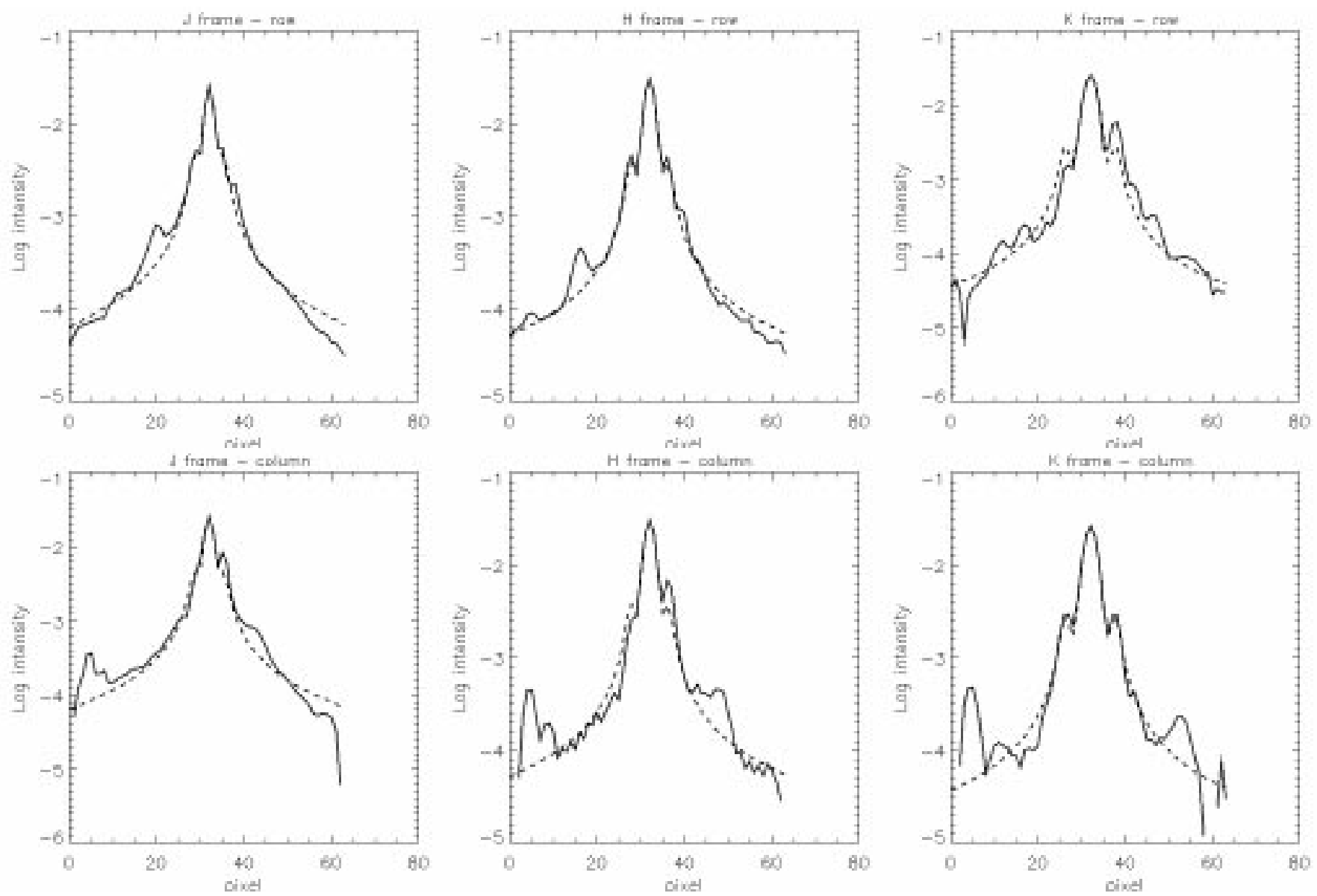

Fig. 6. Comparison of $\mathrm{N}-\mathrm{S}$ (first row) and $\mathrm{E}-\mathrm{W}$ scans (second row) of the detailed mean PSF in the $J, H$ and $K$ bands (solid line) with its radially symmetric approximation (dashed line)

1. the same separation and magnitude difference $\Delta_{\mathrm{m}}$ as in $\tau$ CMa but with different secondary positions (top),

2. the same positions as in $\tau$ CMa but $\Delta_{\mathrm{m}}$ respectively of 1.5, 2.0 and 2.5 (bottom, from left to right).

In both cases the mean PSF in the $\mathrm{H}$ band (Fig. 3, top) was used. These simulated images, in our opinion, sufficiently prove that an expert eye is able to discriminate between an artifact of the AO system and a secondary core reasonably differing (in position and luminosity) from the primary one. But a less subjective way to do this, in dubious cases, is to fit within a small box centered on all the secondary maxima of the image, as was already done in Sect. 2.1 to derive the initial $\sigma$ value. The fit of Eq. (2) to the simulated image set always converges, enabling us to derive the magnitude differences of the components with a standard deviation of about $0.1 \mathrm{mag}$ (for each band, with a systematic decrease from $J$ to $K$ ), and the largest deviations occurring when the secondary is faint and is close to one of the bumps. A second check of the method was done by fitting the good and bad images of Figs. 1 and 2, by taking successively as initial secondary core the three main aberration bumps. Using the brightest bump the fit always converged, restoring the true $\Delta_{\mathrm{m}}$ value within $0.1 \mathrm{mag}$ and the true secondary position within a fifth of a pixel (i.e., $0^{\prime \prime}$ 007), while for the lowest bump the fit diverges or converges to unreliable values, yielding a parametrized image which is clearly different from the observed one and overall with a much smaller correlation $(0.2-0.3)$. To understand the magnitude of the errors (in relative photometry and astrometry) related to the present method, the successive 48 images in each band (see Sect. 1) were analyzed, obtaining results differing from those of Table 2 only by $3-4 \%$ and practically the same standard deviations. This may be considered as an indication that the errors in the procedure are really about half or less of the relative standard deviations, but also as evidence that the atmospheric turbulence was constant, in general, during the image set acquisition. Thus other simulated sets were constructed assuming a binary star model similar to $\tau$ CMa (i.e., with the same magnitude difference 0.9 and the same relative position and separation $0{ }^{\prime \prime} 151$ of the components in each band), while the primary centers were randomly placed within the same pixel, and the same PSF sets of the first simulation in this section were used.

The results from the simulated images, summarized in Table 4 after the rounding off, show that the deviations 

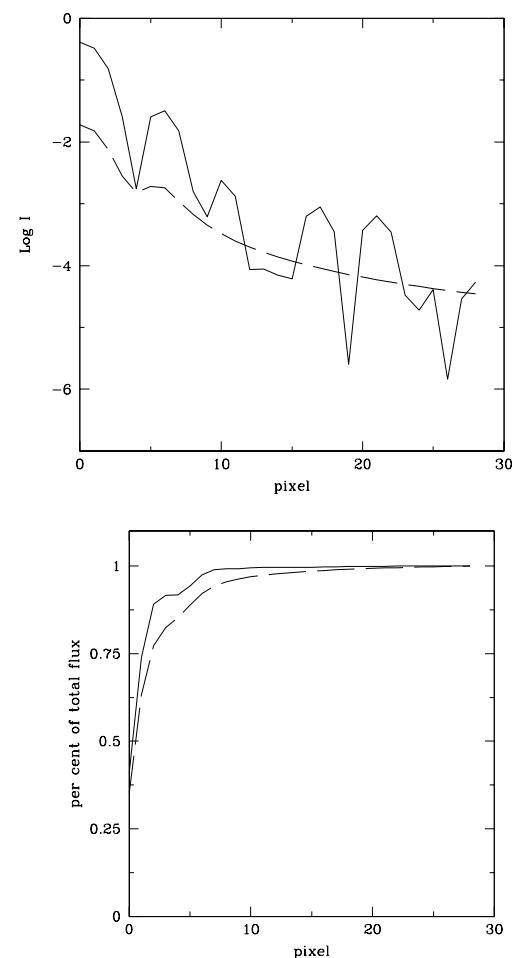

Fig. 7. Global performances of ADONIS during the $\tau$ CMa observations. Top: mean K PSF intensity profile (dashed line) and the diffraction pattern(solid line). Bottom: the related encircled energy

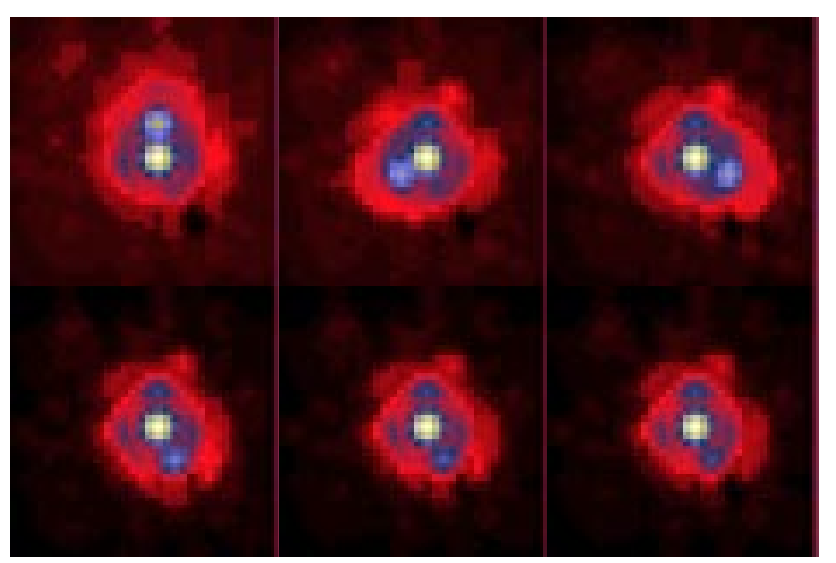

Fig. 8. Appearance of a binary star observed with the partially compensated PSF of ADONIS in the following cases: i) the components magnitude difference and separation is the same as for $\tau \mathrm{CMa}$, but the position angle of the secondary varies (top); ii) the secondary is placed as in $\tau \mathrm{CMa}$, but its magnitude difference is, from left to right, 1.5, 2.0 and 2.5 (bottom images)
Table 4. Simulated image results: i) mean and standard deviations of the individual fits (top) and ii) final values derived from mean images (bottom)

\begin{tabular}{cccc}
\hline & $J$ & $H$ & $K$ \\
\hline$\Delta_{\mathrm{m}}$ & $0.850+0.110$ & $0.950+0.070$ & $0.870+0.060$ \\
& 0.870 & 0.930 & 0.890 \\
$\rho$ & $0.154+0.007$ & $0.150+0.003$ & $0.147+0.004$ \\
& 0.153 & 0.151 & 0.148 \\
\hline
\end{tabular}

from the true values of the estimates of the components' separation and magnitude difference obtained from the mean images are about half of the corresponding ones obtained by averaging the individual fits, and even less of their standard deviations. But the results in Table 4 and those in Tables 2 and 3 have a similar behaviour, allowing us to assume errors of the same order of magnitude also when dealing with real images, i.e., for the final estimates of $\tau$ CMa reported in Table 3. Nevertheless the comparison of results from real and simulated images deserve two further comments. The first is that the standard deviations in Table 2 are calculated by using the mean set values, hence contain a bias component and are larger than those in Table 4, calculated with respect to the true values, i.e., those assumed in doing the simulations. The second refers to the magnitude difference of the components of $\tau \mathrm{CMa}$, which in $\mathrm{H}$ seems larger than in other bands (color effects apart, here not considered), but the simulations also give the same, thus it must be a procedure artifact occurring when the secondary center overlaps the first deformed diffraction ring, as in the present case.

\section{Concluding remarks}

The method assumes that the true photometric structure of the observed source is formed by two shifted and weighted delta functions, and that a set of short PSFs with statistically significant size do not present a marked trend during its acquisition. Also it uses an analytical approximation of the smooth light distribution in the short PSFs to fit short images, obtaining a rough estimate of the photometric parameters but a good determination of the astrometric ones. This allows us to derive by shift-andadd the mean short exposure image, characterized by an improved signal and by the near diffraction-limited resolution, since the image motion of the set images has been removed. Then the mean short image yields by a fit the final adopted features of the binary system and by a deconvolution the detailed mean PSF. The tests and the simulations performed give evidence of the robustness of the present method and of its general capability to resolve Adonis short images of close binary stars with luminosity ratio and relative position of the components spanning a reasonable range; they also enable us to understand the method's 
limitations and the few critical cases, thus strengthening the reliability of the features of $\tau$ CMa reported in Table 3 . It is worth mentioning that the present method, thought to solve a particular problem, may be easily extended to study multiple systems and even crowded stellar fields, provided that in each case the adopted PSF parametrization adopted represents a reasonable compromise between the actual point source image and that corresponding to the full AO compensation, i.e., the diffraction pattern. For instance, dealing with the above quoted images by PUEO (see Sect. 2.1) we must use a globally elongated PSF approximation, with an elliptical core and several confocal elliptical annuli, as will be reported in a future paper. In conclusion, this method of analyzing a set of AO images is fast and robust, and its results are comparable with those obtained by IBD, which is much more time and computer memory consuming. Furthermore, it can run either in an interactive way, as done here, or automatically, since the fundamental steps (search of initial parameters, convergence attainment, image coaddition, agreement or correlation between fitted and observed images and so on) can be controlled by suitable software instructions in an expert program, thus it is amenable to an automatic iterative procedure.

Acknowledgements. The Bologna group would like to thank J. Christou for helpful discussions on IBD and for providing its IDAC code as well as images and other unpublished material. The High Resolution Data Reduction Working Group at ESO is acknowledged for punctual information on its activity and current research efforts. We also thanks David Le Mignan and the $3.6 \mathrm{~m}$ telescope team at La Silla for their help during the observations. We are indebted to G.M. Stirpe for correcting our manuscript and to the referee (E. Thiébaut) whose constructive criticism has much improved the presentation of this paper. This work was supported in part by Ministero della Universitá e della Ricerca Scientifica e Tecnologica (MURST).

\section{References}

Babcock H.W., 1953, PASP 65, 229

Beckers J.M., 1993, ARA\&A 31, 13

Bendinelli O., Parmeggiani G., Zavatti F., 1986, ApJ 308, 611

Bendinelli O., Parmeggiani G., Piccioni A., Zavatti F., 1987, AJ 94, 1095

Christou J.C., Bonaccini D., 1996a, in Technical Report ESO VLT Doc. No. GEN-TRE-ESO-11620-1263

Christou J.C., Bonaccini D., 1996b, in Technical Report ESO VLT Doc. No. GEN-TRE-ESO-11620-1262

Christou J.C., Bonaccini D., 1996c, in Technical Report ESO VLT Doc. No. GEN-TRE-ESO-11620-1261

Christou J.C., Bonaccini D., Ageorges N., 1997, SPIE Proceedings (in press)

Jefferies S.M., Christou J.C., 1993, ApJ 415, 862

Lucy L.B., 1994, in The Restoration of HST Images and Spectra II, Hanisch R.J. and White R.L. (eds.). Space Telescope Science Institute, Baltimore

Penrose R., 1956, Proc. Cambridge Philos. Soc. 52, 17

Rigaut F., Rousset G., Kern P., Fontanella J.C., Gaffard J.P., Merkle F., Lena P., 1991, A\&A 250, 280

Ten Brummelaar T.A., Mason B.D., Bagnuolo W.G., Hartkopf W.I., McAlister H.A., Turner N.H., 1996, AJ 112, 1180

Tessier E., Bouvier J., Beuzit J.L., Brandner W., 1994, The Messenger 78, 35

The Hipparcos and Tycho Catalogues, SP-1200, 1997, ESA, Estec

Thiébaut E., Conan J.M., 1995, J. Opt. Soc. Am. A 12, 485

Tikhonov A.N., Arsenin V.Y., 1977, Solution of Ill-Posed Problems. Winston Pub., Washington DC

Titterington D.M., 1985, A\&A 144, 381

Tyson R.K., 1991, Principles of Adaptive Optics. Academic Press Inc., New York

Véran J.P., Rigaut F., Maître H., Rouan D., 1997, J. Opt. Soc. Am. A 14, 3057 